\title{
Paris MEM: a study protocol for an effectiveness and efficiency trial on the treatment of traumatic stress in France after the 2015-16 terrorist attacks
}

\author{
A. Brunet ${ }^{1,2}$, A. Ayrolles 1,3 , L. Gambotti ${ }^{3}$, R. Maatoug ${ }^{1,3}$, C. Estellat ${ }^{3}$, M. Descamps ${ }^{1,2}$, N. Girault ${ }^{1,3}$, K. Kalalou ${ }^{4}$, \\ G. Abgrall ${ }^{5}$, F. Ducrocq ${ }^{6}$, G. Vaiva ${ }^{6}$, N. Jaafari ${ }^{7}$, M. O. Krebs ${ }^{8}$, E. Castaigne ${ }^{9}$, I. Hanafy ${ }^{10}$, M. Benoit ${ }^{11}$, S. Mouchabac ${ }^{12}$, \\ M. C. Cabié ${ }^{13}$, O. Guillin ${ }^{14,15}$, F. Hodeib ${ }^{1,3}$, I. Durand-Zaleski ${ }^{16}$ and B. Millet ${ }^{1,3^{*}}$ (D)
}

\begin{abstract}
Background: The Paris and Nice terrorist attacks affected a thousand of trauma victims and first-line responders. Because there were concerns that this might represent the first of several attacks, there was a need to quickly enhance the local capacities to treat a large number of individuals suffering from trauma-related disorders. Since Reconsolidation Therapy (RT) is brief, relatively easy to learn, well tolerated and effective, it appeared as the ideal first-line treatment to teach to clinicians in this context.

Methods: This study protocol is a two-arm non-randomized, multicenter controlled trial, comparing RT to treatment as usual for the treatment of trauma-related disorders. RT consists of actively recalling one's traumatic event under the influence of the ß-blocker propranolol, once a week, for 10-25 min with a therapist, over 6 consecutive weeks. This protocol evaluates the feasibility, effectiveness, and cost-utility of implementing RT as part of a large multi-center $(N=400)$ pragmatic trial with a one-year follow-up.

Discussion: Paris MEM is the largest trial to date assessing the efficiency of RT in the aftermath of a large-scale man-made disaster. RT could possibly reinforce the therapeutic arsenal for the treatment of patients suffering from trauma-related disorders, not only for communities in western countries but also worldwide for terror- or disasterstricken communities.
\end{abstract}

Trial registration: Clinical Trials (ClinicalTrials.gov). June 3, 2016. NCT02789982.

Keywords: Post-traumatic stress disorder (PTSD), Terrorism, Reconsolidation, Cost-effectiveness trial

\section{Background}

The terrorist attacks that struck France in 2015 and 2016 were the most devastating and deadly events to occur on its territory since WWII, with a thousand of individuals physically or psychologically injured by the attacks according to official numbers [1]. Past research suggest that up to $20 \%$ of individuals exposed to this

\footnotetext{
* Correspondence: b.millet@aphp.fr

'Département de Psychiatrie adulte, boulevard de I'Hôpital, 75013 Paris, France

${ }^{3}$ Département de Psychiatrie adulte, Hôpital Universitaire de la Pitié Salpêtrière, Assistance Publique - Hôpitaux de Paris, boulevard de l'Hôpital, 75013 Paris, France

Full list of author information is available at the end of the article
}

type of event develop posttraumatic stress disorder (PTSD) [2]. PTSD's core symptoms are: re-experiencing, avoidance, alterations in mood and cognition, and hyperarousal [3]. Epidemiological studies indicate a median time of 3 years before remission when the disorder is treated, and of 5 years when left untreated, with a significant proportion of unremitted individuals 10 years after trauma exposure [2]. PTSD is also frequently comorbid with major depression (48-55\%), dysthymia (21-23\%), generalized anxiety disorder (15-17\%), social phobia (28\%), specific phobia (29-31\%), panic disorder (7-13\%), alcohol abuse (28-52\%), and substance abuse (27-35\%) $[2,4,5]$. Given the magnitude of the events that took 
place in France in 2015-16, and considering the poor prognosis of PTSD from a human suffering perspective, in the aftermath of the terrorist attacks there was an urgent need to quickly enhance the treatment resources in France. There were also fears that this attack might be the first one of a series of such events. We also reasoned that this massive terrorist attack might serve as a learning experience and, if successful, the treatment strategy deployed in Paris and Nice might serve in similar circumstances elsewhere in the future.

In order to address this unexpected plight, the research center of the Douglas Mental Health University Institute (Canada) and the public healthcare system of Paris (Assistance Publique - Hôpitaux de Paris [AP-HP], University Hospitals of Ile de France, France) worked together to build an innovative care proposition, named Paris MEM (shorthand for 'Paris, Mémoire Vive'). The aims of this project were: (a) to quickly train a group of at least 40 mental health professionals on 20 sites in using Reconsolidation Therapy (RT), as developed by Alain Brunet [6]; (b) to offer to patients, in addition to the already existing care propositions (i.e., treatment as usual), a brief innovative first line treatment for PTSD; (c) to assess the effectiveness and the cost-utility of RT versus treatment as usual (TAU), and (d) to enhance the overall preparedness in France, in the event of subsequent large scale terrorist attacks. In this context, RT appeared as an appealing treatment modality since it is brief, well tolerated and effective [7] and its implementation seemed relatively easy.

\section{Reconsolidation as an emerging therapy for PTSD}

Upon retrieval, under conditions of mismatch, a consolidated memory returns to a labile state before undergoing re-consolidation [8-10]. A large body of animal and human research shows that a deconsolidated memory can be impaired after its reactivation using a pharmacological agent (for a review see $[11,12]$ ), notably with the $\beta$-adrenergic receptor blocker propranolol (for a review see $[13,14])$. Propranolol is a lipophilic $\beta$-blocker, widely used for a variety of diseases in the last 50 years, with very few contraindications and side effects.

In 2008, Brunet et al. [15] were the first to show in a randomized controlled trial that long-standing traumatic memories can be durably toned down, using a treatment protocol congruent with reconsolidation theory. This intervention protocol was further refined and tested in three independent open-label trials in France, Canada and the United States [6]. The results showed a significant posttreatment decrease of PTSD symptoms, as well as at the 6-month follow-up. A recent randomized controlled trial comparing RT versus trauma reactivation + placebo confirmed such findings [7]. Reconsolidation impairment using propranolol has also been used with success in small randomized protocols for the treatment of phobias [16] and addiction [17]. In its current form, the treatment protocol for PTSD requires six once-aweek sessions of $10-25$ min each with a therapist.

\section{Current treatments for PTSD}

Psychotherapies such as Cognitive-Behavioral Therapy (CBT) and Eye Movement Desensitization and Reprocessing (EMDR), and pharmacological interventions such as the Selective Serotonin Reuptake Inhibitors (SSRIs) are all among the recommended treatment modalities for PTSD according to most guidelines $[14,18-21]$ and according to a Cochrane review [22]. In three randomized controlled trials $(N=1070)$, paroxetine had a significantly higher response rate than placebo ranging from 29 to $67 \%$ after 12 weeks [23-25]. The mean time for response ranged between 4 and 6 weeks. SSRIs such as paroxetine, while effective in the treatment of PTSD do not, however, offer a cure for PTSD. They must be taken daily for at least 1 year, and their side effects often lead patients to discontinue them [26]. CBT, including EMDR, comprise a variety of related approaches that have at their core exposure and/or cognitive restructuring as their main therapeutic ingredient $[27,28]$. The recommended duration of individual therapy is 6-12 ninety-minutes sessions of treatment, offered once or twice weekly in an outpatient setting [14,27]. CBT and EMDR both require strong educational and clinical backgrounds and can hardly be quickly learned in the aftermath of mass trauma. Furthermore, of the $2 / 3$ of those who benefit from CBT at post-treatment, half of them relapse within 1 year, thereby reducing the treatment's efficiency [29]. For all those reasons, we opted to teach RT therapy to the clinicians participating in this trial.

\section{Efficacy, effectiveness and efficiency}

As summarized by Kim [30], efficacy is the extent to which an intervention works under ideal circumstances while effectiveness assesses whether an intervention works when provided under usual circumstances of healthcare practice. Research on effectiveness is also described as pragmatic trials (see Additional file 1) [31]. Pragmatic trials have several characteristics: 1) patient eligibility and recruitment should reflect routine practice; settings should reflect the care facilities available to a diverse population; 2 ) the intervention should be provided by normal staff with routine training; 3) intensity of follow-up should be similar to typical follow-up in usual care; 4) primary outcome should be directly relevant to participants and all patients should be included in the analysis of the primary outcome; 5) outcome measures are patient-centered and incorporate broad measures of health. Finally, efficiency refers to the ability to achieve a therapeutic goal using a lesser amount of time 
and resources than what is typically observed. Typically, efficiency is assessed by cost-utility analysis.

The co-primary aims of this pragmatic trial are to evaluate the effectiveness and the efficiency of RT therapy, as deployed in a post-terrorist attack real-world setting. Specifically, we wish to explore the feasibility of implementing in routine practice an innovative treatment for PTSD on a large scale and in a short period of time; to evaluate the acceptability of the treatment by the clinicians and from the patients' perspectives; to assess the efficacy of RT; to determine its cost-utility compared to treatment as usual (TAU) over a 1-year period.

\section{Methods}

\section{Study design}

This study is a two-arm non-randomized, multicenter controlled trial, comparing RT to TAU for the treatment of trauma-related disorders. As shown in Table 1, psychometric assessments are made at baseline, after 6 weeks of treatment, as well as 3 and 12 months following study inclusion. In order to enhance external validity this study protocol is implemented into the usual healthcare system in France. Patients are offered the choice of the intervention they wish to receive. The study protocol was approved by local ethics committee (Comité de protection des personnes for Ile de France VI \#CPP/14-16), and regulatory agencies (Commission nationale de l'informatique et des libertés \#GPR1713292F and Agence nationale de sécurité du medicament. We plan to include up to 400 participants with the possibility of stopping at the end of the pre-specified inclusion period if at least 200 receiving $\mathrm{RT}$ are enrolled.

\section{Eligibility criteria}

Participants will be at least 18 years old without any upper age limit. Eligible participants must read and speak French, have a DSM-5 diagnosis of PTSD, adjustment disorder or any other specified trauma-related disorder. They must have a symptom score above 43 on the PTSD Checklist [32] and be at least moderately ill according to the Clinical Global Impression scale. They must not have a severe mental or neurological disorder (i.e., a history of bipolar or psychotic disorder, acute suicidal, homicidal, or self-injuring intentions, a traumatic brain injury in the last 5 years, an alcohol or substance dependence disorder), nor be involved in trauma-related litigation. Pregnant or breastfeeding women cannot participate in the trial for security reasons. In addition, to participate in the RT group, patients taking psychotropic medications must be on a stable dosage for at least 2 months, they must not use any medications that involve a dangerous interaction with propranolol, or have a resting heart rate inferior to 55 beats per minute, or a resting systolic blood pressure below $100 \mathrm{mmHg}$; any other contraindicating medical condition, as determined by the study physician (Fig. 1).

\section{Procedure}

Patients presenting with trauma-related symptoms to participating mental healthcare centers in Paris (15 centers), Lille, Fort-de-France, Poitiers and Nice (2 centers)

Table 1 Overview of measures and time of assessment

\begin{tabular}{|c|c|c|c|c|c|c|c|c|c|c|c|}
\hline \multirow[t]{2}{*}{ Instrument } & \multirow[t]{2}{*}{ Variable } & \multirow[t]{2}{*}{ T0 } & \multicolumn{6}{|c|}{ Treatment sessions } & \multirow[t]{2}{*}{$\mathrm{T} 1$} & \multirow[t]{2}{*}{$\mathrm{T} 2$} & \multirow[t]{2}{*}{$\mathrm{T} 3$} \\
\hline & & & t1 & t2 & t3 & t4 & t5 & t6 & & & \\
\hline Demographics & Sociodemographic characteristics & $X$ & & & & & & & & & \\
\hline PCL-S & PTSD symptom severity & $x$ & $x$ & $x$ & $x$ & $x$ & $x$ & $x$ & $x$ & $x$ & $x$ \\
\hline CGl & Global patients' condition & $*$ & $x$ & $x$ & $x$ & $x$ & $x$ & $x$ & * & * & $*$ \\
\hline MINI-S & Diagnosis and comorbidities & $x$ & & & & & & & $x$ & $x$ & $x$ \\
\hline PDI & Severity of trauma exposure & $X$ & & & & & & & & & \\
\hline PDEQ & Peritraumatic dissociation & $X$ & & & & & & & & & \\
\hline WHOQOL-BREF & Quality of life & $X$ & & & & & & & $x$ & $x$ & $x$ \\
\hline$E Q-5 D-5 L$ & Quality of life & $X$ & & & & & & & $x$ & $x$ & $x$ \\
\hline MEDEC & Health service utilization & $X$ & & & & & & & $x$ & $x$ & $x$ \\
\hline $\mathrm{HSCL}-25$ & Anxiety and depressive symptoms & $X$ & & & & & & & $x$ & $x$ & $x$ \\
\hline QFS & Social functioning & $x$ & & & & & & & $x$ & $x$ & $x$ \\
\hline
\end{tabular}

*Assessed by a clinician blind to the patient's treatment modality

TO: baseline assessment

T1: posttreatment assessment (week 7)

T2: 3-month follow-up assessment

T3: one-year follow-up assessment

PCL-S: PTSD Checklist - Specific Version, CGI: Clinical Global Impression, MINI-S: Mini International Neuropsychiatric Interview for DSM-5, PDI: Peritraumatic Distress Inventory, PDEQ: Peritraumatic Dissociative Experiences Questionnaire, WHOQOL-BREF: World Health Organization Quality of Life (brief), EQ-5D-5 L: EuroQol

Questionnaire, MEDEC: Medico-Economic Questionnaire, HSCL-25: Hopkins Symptom Checklist, QFS: Social Functioning Questionnaire 


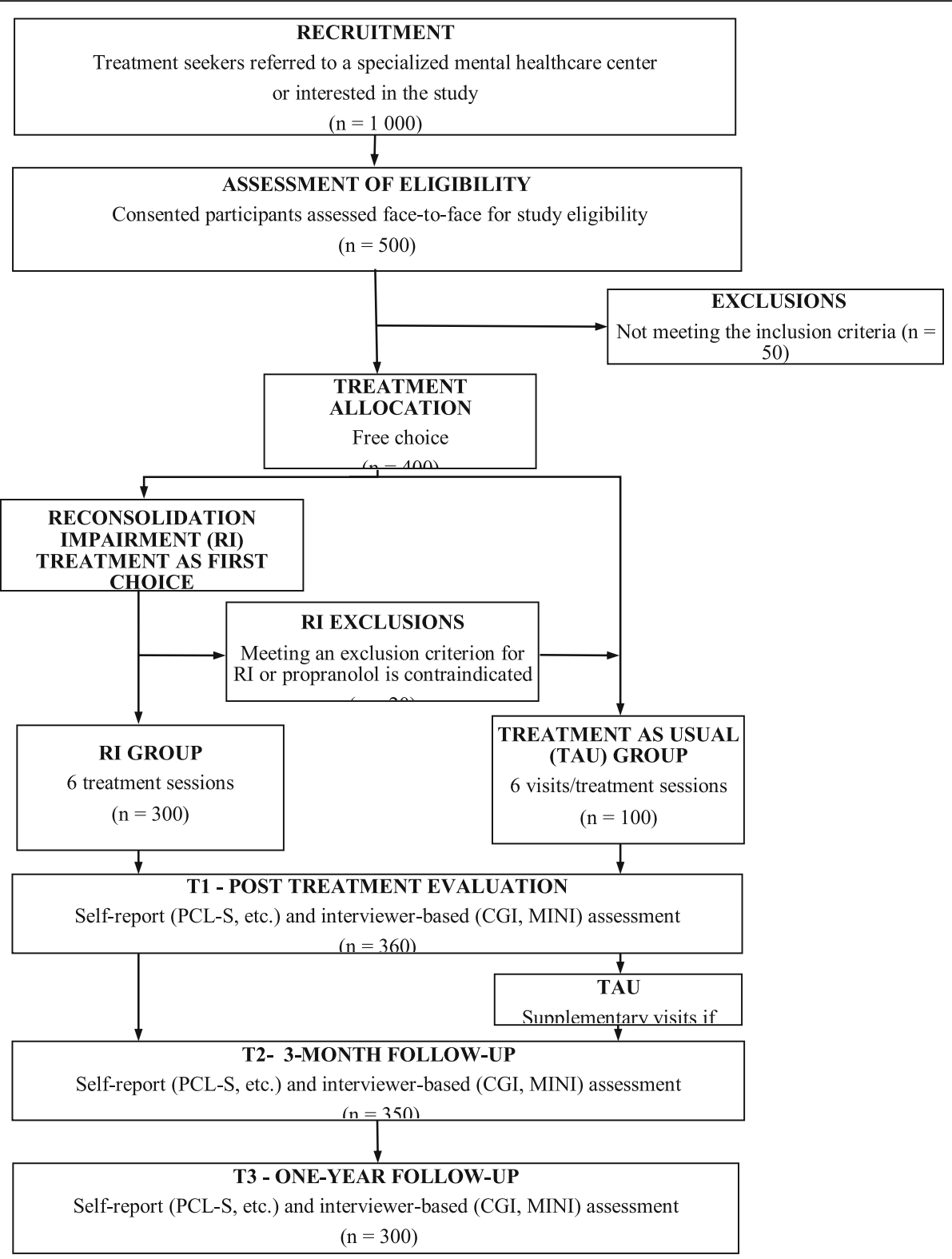

Fig. 1 Study flowchart

regions are informed of the study. After the study has been fully explained to them, interested patients are asked to sign the informed consent form. The clinician then formally investigates if the study requirements are fulfilled and determines patient eligibility. Based on the patients' choice and their eligibility for the RT group, enrolled patients are then allocated to RT or TAU. The treatment begins within 7 days of enrolment. A series of six treatment sessions are set on a once a week basis, but with a margin of 4-15 days between sessions. The patients' general practitioner is informed about their patient's participation in the study. The symptom assessments are conducted for both groups at baseline, and then 7,13 , and 52 weeks after study inclusion.

\section{Clinicians training}

A two-day course is provided to the participating clinicians by the first author (A.B.). Clinicians are either medical doctors with a mental health background or practice, practicing residents and psychiatrists, or practicing psychologists. The first part of the training involves the proper identification of trauma-related disorders. The second part reviews the empirical evidence in favor of RI. The third part is practical and addresses propranolol prescription rules, role playing, RT procedures (see also Brunet et al., 2018) and research protocol information. Online clinical supervision is offered to each clinician via a private forum moderated by 
A.B. as well as personalized telephone support with A.B. or B.M. regarding specific questions, on an as needed basis. Treatment adherence is monitored in the following way: selection of the index traumatic event, performance of a cardiogram (first session only), oral propranolol dosage relative to ideal body mass, ingestion of a light snack with the propranolol medication, heart rate and blood pressure at baseline and, following ingestion of propranolol, the participant's side effects to propranolol, the time elapsed between propranolol ingestion and the beginning of the treatment session, the completion of a self-report PTSD symptom measure by the patient covering the previous week, the length (number of pages) of the trauma narrative, use of the first-person singular, and verb tense (present) of the trauma narrative, the inclusion in the trauma narrative of five or more descriptors (from a list) of peritraumatic physical reaction, whether the patient actually read aloud his or her full script, the designation to the therapist of the hot spot in the trauma narrative, the possibility to revise/ supplement the trauma narrative at each treatment visit, the number (six) and duration (10-25 min.) of the treatment visits, the number of days between each treatment visit, as well as the lack of use of any other empiricallyvalidated treatment method for PTSD by the treating clinician during the treatment sessions.

\section{Study treatments}

Reconsolidation Therapy ${ }^{\mathrm{mm}}(\mathrm{RT})$ consists in actively recalling one's index (i.e. worst/traumatic) event, using a specific memory reactivation protocol, under the influence of the $ß$-blocker propranolol, once a week, for 10-25 min with a therapist, over 6 consecutive weeks. More specifically, $1 \mathrm{mg} / \mathrm{kg}$ of oral propranolol (Inderal ${ }^{\mathrm{mm}} /$ Avlocardyl $^{\mathrm{Tm}}$ ) based on ideal body mass index (BMI) is given at the treatment center $75 \mathrm{~min}(+/-15 \mathrm{~min})$ before the session. Once the session begins, the patients are asked to write (session 1) and read out-loud (sessions 1-6) a 1-2 pages narrative of their traumatic event. On subsequent sessions, the patients are asked if they wish to update or modify their narrative to include new/more detailed information thatg bothered them during the previous week. The trauma narrative, a description of the event and of the trauma's hot spot (i.e. the most bothersome/unacceptable part of the event), includes at least five bodily sensations and is written in the first person singular, present tense [15].

Treatment as usual (TAU) involves receiving the PTSD treatment offered locally at this treatment center, whatever this treatment may be. In most centers, TAU was an SSRI (most commonly, paroxetine 20-60 mg started at the first treatment visit), and/or a form of psychotherapy, typically CBT, EMDR, supportive therapy or psychodynamic therapy [26-28].

\section{Instruments}

The primary outcome for the effectiveness analysis is the difference between the group mean PTSD ChecklistSpecific (PCL-S) score at inclusion (T0) minus the same score obtained at the one-year follow-up (T3). The PCL$\mathrm{S}$ will be administered at baseline (T0), at each treatment session (t1-t6) and 1 week posttreatment (T1), 3-month follow-up after baseline (T2) and one-year follow-up after baseline (T3).

The reliable and valid PCL-S $[32,33]$ is a 17 -item selfreport scale that assesses DSM-IV-TR PTSD symptoms in the past week from the perspective of the patient. (No validated French PCL version compatible with the DSM5 was available when the study began). The PCL-S ranges between 17 (no symptom) and 85 (maximum score). For study inclusion, a cut-off score of equal or greater than 44 is used, as done by others [34, 35] (Fig. 2).

The Clinical Global Impression (CGI) scale is used as a global evaluation of the patients' condition and symptom evolution. The CGI is composed of two parts: illness severity (CGI-S) and improvement (CGI-I), each of which is rated on a 7-point scale where 1 represents health (not ill/very much improved) and 7 represents illness (extremely ill/much worse). The CGI is administered by a clinician blind to the patient's treatment modality.

PTSD or adjustment disorder diagnosis and comorbidities, are assessed with the MINI International Neuropsychiatric Interview (MINI-S), a structured diagnostic interview based on the DSM-5. PTSD, adjustment disorder, anxiety modules as well as lifetime and current depression, suicidality, obsessive-compulsive disorder, alcohol and substance abuse and dependence are assessed in order to report any comorbid disorders that are frequently associated with PTSD. The MINI-S is the new version of the MINI interview [36]; its validation in French is in progress at our center and elsewhere.

Distress and dissociation at the time of trauma are assessed with the reliable and valid Peritraumatic Distress Inventory (PDI) $[37,38]$ and the Peritraumatic Dissociative Experiences Questionnaire (PDEQ) [39, 40], respectively. The 13-item self-report PDI documents the recalled emotional responses experienced at the time of the trauma and, as such indexes the subjective severity of the trauma from the patient's perspective. It ranges between 0 (no symptom) and 52 [41]. The 10-item selfreport PDEQ measures the recalled dissociative reactions experienced during or immediately after the traumatic event and is an index of poor prognosis. Items are scored from 1 to 5 with a total score ranging between 10 (no symptom) and 50.

The primary outcome for efficiency (cost-utility) analysis is Quality of life at 12 months assessed with the 


\section{Confidence intervals}

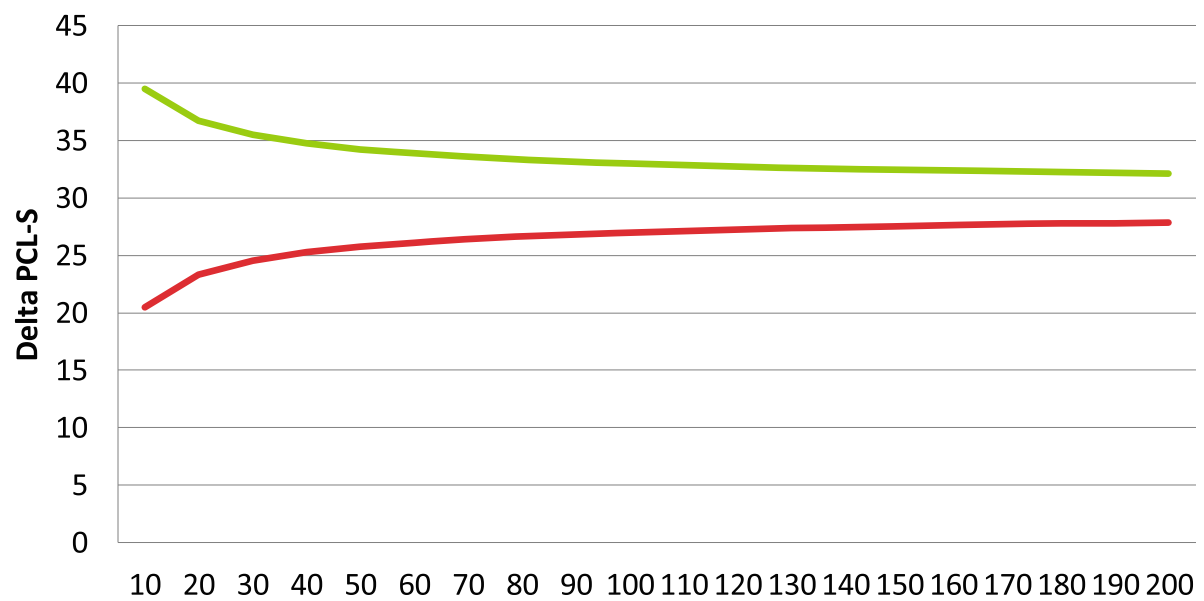

Fig. 2 Evolution of the confidence interval according to the sample size and the delta PCL-S (green line: delta PCL-S above 30; red line: delta PCL-S below 30)

reliable and valid EuroQol questionnaire (EQ-5D-5 L) [42, 43]. The EQ-5D-5 L assesses five health dimensions: mobility, self-care, usual activities, pain/discomfort and anxiety/depression. Each dimension is rated on five levels of severity [42, 43]. The utility value set for each score is available for the French population. These values will be used in the assessment of efficiency of RT. We will also use the 26-item self-report World Health Organization Quality of Life WHOQOL-Bref [44, 45], which measures the extent to which illness impairs the subjective wellbeing of patients at the time of the assessment. Each item scored 1-5 is summed and transformed into a 0 (poorest QOL) to 100 (best QOL) symptom score. The scale produces four main scores: physical, psychological, social relationship and environment.

Costs related to healthcare utilization and work status are evaluated with the MEDico-EConomic Questionnaire (MEDEC) adapted from the Client Service Receipt Inventory $[46,47]$ in order to meet the specificity of the French health system. This inventory collects retrospective information on health service utilisation in order to estimate illness related costs. It includes a history of hospitalization, sick leave, treatments received, medical consultations, as well as all paramedic consultations regardless of the reason.

This economic evaluation is conducted in accordance with the recommended methods of the HAS (Haute Autorité de Santé or French Health Authority) and the CHEERS (Consolidated Health Economic Evaluation Reporting Standards) [48].

Social functioning is evaluated with the Social Functioning Scale (QFS) [49]. The 16-item self-report QFS assesses the frequency and satisfaction with a number of social behaviours in a psychiatric adult population. Each item is scored from 1 to 5 with frequency and satisfaction scales ranges between 8 and 40 and a total range of 16 to 80 over the previous 2 weeks and is sensitive to change after treatment [49].

General anxiety and depressive symptoms are evaluated using the 25-item self-report Hopkins Symptom Checklist (HSCL-25) [50], which includes a subscale measuring anxiety and another measuring depression (each item is scored 1-4, and the total mean score also ranges from 1 to 4 , with the clinical cut-off being set at $1,75)$. The total score is correlated with emotional distress of unspecified diagnosis, and the depression score is correlated with major depression as defined by the DSM-IV-TR [51].

\section{Statistical analyses}

The clinical scores will be analyzed using an intention to treat (ITT) analysis: all patients allocated to RT or TAU will be analyzed. The collected data will be used to describe the 2 cohorts: the RT group and the TAU group. Variables will comprise socio-demographic parameters, duration of PTSD, psychiatric comorbidities, pre-morbid disorders, and co-prescriptions. As described above, the primary outcome for effectiveness analysis is based on the delta (T3 minus T0) of PTSD symptom severity (PCL-S). A first indicator will be the mean PCL- S difference between $\mathrm{T} 3$ and T0. The delta of EQ5D-5 L (T3 minus T0) examines efficiency from the health economic perspective.

A regression model will be used to calculate a second indicator based on the PCL-S values (t1-t6) collected between T0 and T1, allowing for the evaluation of PTSD symptoms evolution over time and its variation between individuals. A third indicator will be the rate of patients 
considered as treatment responders (PCL-S score $<44$ ) at Week 7 (T1), Week 13 (T2) and Week 52 (T3). Mean and SD will also be computed for the delta of CGI score between T1 and T0. Missing data will be replaced using multiple imputation methods, provided that the data is missing at random or completely at random.

Additionally, in order to take into account the difference between the features of both cohorts (RT and TAU), we will use a propensity score of being allocated to RT to adjust the different model. For the secondary criteria, these propensity scores will be applied to the other scales used for the assessment.

Costs will be estimated from the point of view of society for the follow up period (1 year) without discounting, due to the study duration. We will estimate in each group the median cost, mean and $95 \%$ confidence interval $(95 \% \mathrm{CI})$ using bootstrap replications because of distribution skewedness and calculate the cost difference. Health-related QOL is collected using the EQ-5D-5 L self-administered questionnaire at baseline, at 3 months and at 1 year. The utility values will be based on French tariffs. The efficacy of the intervention will be estimated by quality adjusted life years (QALYs), using the EQ-5D$5 \mathrm{~L}$ scores and the time between treatment sessions. The difference in QALYs is the difference in the area between the utility curves for the two groups. Between-group comparisons of QALYs and costs will be performed with the appropriate statistical tests for their distribution, with a significance threshold of .05 (two-sided test).

The incremental cost utility ratio is the difference in average total costs divided by the difference in average total QALYs. The joint comparison of costs and effects will use nonparametric bootstrapping with 1000 resamples and generate the scatterplot on the cost effectiveness plane as a well as an acceptability curve.

\section{Sample size}

This study aims to assess the usefulness of RI. The number of patients should be considered as a continuous variable, which contributes to the precision of the estimates of the results: -Means, course secondary to treatments, based on anterior studies using the delta (Day 1 - week 7) on the PCL-S score. One should expect a pre -/post-treatment PCL-S difference of 30 points with a SD of 15-25 points [7]. If a symptom reduction of 5-9 points on the PCL-S represents a favorable response and a reduction greater than 10 represents a clinically meaningful reduction, one should expect that $70 \%$ of patients display a favorable response to the RT treatment [7]. Based on those results, the confidence intervals will evolve according to the plot proposed below. For example, a 95\%CI calculated over 200 patients will vary between 27 and 33.

\section{Discussion}

The fight against terrorism should involve all mental health specialties. To fulfill that role, effective and innovating multimodal treatments are required. At its conclusion, Paris MEM will become the largest pragmatic trial to date assessing whether RT works under the usual healthcare practice that prevails in the aftermath of a large-scale man-made disaster (effectiveness). Paris MEM aims to determine if RT can become the preferred first-line therapeutic intervention for traumatic stress following a wide scale catastrophic event, considering that it is easy to teach to clinical staff and may treat trauma-related disorders using a lesser amount of time and resources than TAU (efficiency). RT could reinforce the therapeutic arsenal for the treatment of PTSD patients, not only for communities in western countries but also worldwide for terror- or disaster-stricken communities who wish to undo some of the psychological damage they sustained.

One of the aims of the Paris MEM study is to provide an educational phase on an innovative therapeutic method able to help practitioners of the various healthcare institutions of Paris to treat PTSD. While building the experimental design, one of our main concerns was the feasibility and acceptability of the treatment procedure from the therapists' and patients' perspectives. As mentioned in the Methods section, one of the main outcomes will combine cost and effectiveness 1 year following the treatment. In that sense, we aim for a noninferiority procedure compared to TAU, hoping for a better cost-utility outcome of the RT method.

The absence of randomization could be considered a limitation. However, this choice is in line with the choice of a pragmatic trial, and ensures feasibility, considering that -based on our prior experience- we expect that patients will express a preference for RT compared to TAU. Due to the extraordinary circumstances in which this study was initiated, we felt that it would be wiser to conduct the trial by asking the study participants to express their preference toward the treatment they wished to receive (RT or TAU). Ultimately, the choice of treatment of the participants will inform us as to the treatment acceptability of RI. Additionally, recruitment would be easier using a free choice approach. As mentioned previously, a regression model highlighting the course of PTSD symptom severity over time will allow us to measure the proportion of patients responding to treatment and achieving remission. The comparison of the two treated cohorts will involve a propensity score of choosing RT treatment, in order to take into account measurable confusion factors and compensate for the absence of randomization. Also, to limit selection bias, eligibility criteria are the same for the two groups, except for contra-indications to propranolol. In the context of 
the terrorist attacks and ensuing mass suffering, and given the existence of efficacious treatments (SSRIs or psychotherapies), this effectiveness study is not compatible with the implementation of a placebo arm. A placebo controlled trial was recently published and showed the efficacy of RT versus placebo [7].

The absence of blinding is another limitation in our study. Neither the patients nor the assessors are blind for the primary criterion of assessment (PCL-S score), as is always the case in psychotherapy research. A singleblind pre/post evaluation is conducted for the CGI criterion, with the same rater who does not participate in the patient's care. In addition, because the main outcome is a PTSD symptom self-report -and not a clinician-based interview- there is no interviewer bias.

\section{Conclusion}

Paris MEM was initiated in the context of the most severe attacks to occur in France since WWII. This study illustrates the importance given by the French health authorities and the AP-HP, to the consequences of acute stress in the context of terrorism. RT represents an innovative treatment that could prove to be the most costeffective treatment proposed in this domain up to this day. We strongly believe that Paris MEM will increase our understanding of how to effectively alleviate post-traumatic suffering in the aftermath of mass traumatization, and that it has the potential to serve as an inspiring example to the world.

\section{Supplementary information}

Supplementary information accompanies this paper at https://doi.org/10. 1186/s12888-019-2283-4.

Additional file 1. Nine Dimensions for Assessing the Level of Pragmatism in a Trial, as Proposed in the Pragmatic-Explanatory Continuum Indicator Summary 2 (PRECIS-2) Tool (Ford I, Norrie J. 2016) [31].

\section{Abbreviations}

CBT: Cognitive-Behavioral Therapy; CGI: Clinical Global Impression; CHEERS: Consolidated Health Economic Evaluation Reporting Standards; EMDR: Eye Movement Desensitization and Reprocessing; EQ-5D-5 L: EuroQol Questionnaire; HAS: Haute Autorité de Santé or French Health Authority; HSCL-25: Hopkins Symptom Checklist; ITT: Intention to treat; MEDEC: MEDicoEConomic Questionnaire; MEDEC: Medico-Economic Questionnaire; MINIS: Mini International Neuropsychiatric Interview for DSM-5; PCL-S: PTSD Checklist - Specific Version; PDEQ: Peritraumatic Dissociative Experience Questionnaire; PDI: Peritraumatic Distress Inventory; PTSD: Post traumatic disorder; QALYS: Quality adjusted life years; QFS: Social Functioning Questionnaire; RT: Reconsolidation therapy; TAU: Treatment as usual; WHOQOL-BREF: World Health Organization Quality of Life (brief); WHOQOLBref: World Health Organization Quality of Life instrument

\section{Acknowledgements}

We sincerely thank the centers who participated in the Paris MEM study: Pitié Salpêtrière Hospital (Dr Girault); Ville Evrard Hospital (Dr Januel), Tenon Hospital (Dr Abgrall), Corentin Celton Hospital (Dr Louville), Lille University Hospital (Dr Ducroca and Pr Vaiva), Nice University Hospital (Pr Benoit), Marne La Vallée Hospital (Dr Marc), Sainte-Anne Hospital (Pr Krebs), Bicêtre University Hospital (Pr Hardy), Saint-Antoine University Hospital (Dr
Mouchabac), Poitiers University Hospital (Pr Jaafari), Saint Maurice Hospital (Dr Cabié), and Rouen University Hospital (Pr Guillin). We thank Deniker Foundation for its supportive network. We particularly wish to thank the AP-HP Assistance public - Hôpitaux de Paris for supporting this work.

\section{Authors' contributions}

$A B, B M$ and IDZ contributed to the study conception and design, analytical plan. $A B, B M, I D Z, L G, A A, M D$ and $F H$ drafted the manuscript and contribute to and critical revision of the manuscript. BM, AA, MD, NG, KK, GA, FD, GV, $\mathrm{NJ}, \mathrm{MOK}, \mathrm{EC}, \mathrm{IH}, \mathrm{MB}, \mathrm{MCC}, \mathrm{SM}, \mathrm{FD}, \mathrm{OG}$ and FH contributed to data acquisition. RM and $C E$ have responded to the reviewers comments. All authors have read and approved the final version of the manuscript.

\section{Funding}

The study has been funded by Assistance Publique - Hôpitaux de Paris, Fondation Assistance Publique - Hopitaux de Paris and MSD Avenir. The study was independently reviewed by the funding committee.

\section{Availability of data and materials}

The datasets used and/or analyzed during the current study belong to the AP-HP (Assistance Publique - Hopitaux de Paris) research directorate and will be available by request to the corresponding author.

\section{Ethics approval and consent to participate}

This study has been performed in accordance with the Declaration of Helsinki and have been approved protocol was approved by Ethics committees in France (Comité de protection des personnes for lle de France VI (\#CPP/14-16).) Written informed consent will be obtained from all patient prior to participation to the study.

\section{Consent for publication}

All data included in this manuscript received consent for publication.

\section{Competing interests}

The authors declare that they have no competing interests.

\section{Author details}

'Département de Psychiatrie adulte, boulevard de l'Hôpital, 75013 Paris, France. ${ }^{2}$ Department of Psychiatry, McGill University, Montréal, Canada. ${ }^{3}$ Département de Psychiatrie adulte, Hôpital Universitaire de la Pitié Salpêtrière, Assistance Publique - Hôpitaux de Paris, boulevard de l'Hôpital, 75013 Paris, France. ${ }^{4}$ Unité de recherche clinique, EPS de Ville Evrard, G03, 5 rue du Dr Delafontaine, 93200 Saint-Denis, France. ${ }^{5}$ Assistance Publique Hôpitaux de Paris -, Hôtel-Dieu, 75004 Paris, France. ${ }^{6}$ France CHRU de Lille, Pôle de Psychiatrie Médecine Légale et Santé en milieu Pénitentiaire, SCA-Lab CNRS UMR 9193, 59037 cedex Lille, France. ${ }^{7}$ CIC INSERM U802, CHU de Poitiers, Unité de recherche clinique intersectorielle en psychiatrie du Centre Hospitalier Henri Laborit, 86022 Poitiers, France. ${ }^{8}$ Centre Hospitalier Sainte Anne, Service Hospitalo-Universitaire, Faculté de Médecine Paris Descartes, Université Paris Descartes, Paris, France. ${ }^{9}$ Service de Psychiatrie, CHU de Bicêtre, HUPS, APHP 78, rue du général Leclerc, 94270 Le Kremlin Bicêtre, France. ${ }^{10} \mathrm{CH}$ Marne La Vallée, Service de Médecine Légale, 77420 Marne-La-Vallée, France. ${ }^{11}$ Clinical Neuroscience Department Hospital Pasteur 1, France University of Côte d'Azur, 30 avenue de la voie, 06002 NICE cedex 1 Romaine, France. ${ }^{12}$ Département de psychiatrie et de psychologie médicale de l'adulte, Hôpital universitaire Saint-Antoine, Université Pierre et Marie Curie, Paris VI - AP-HP, 184 rue du Faubourg-Saint-Antoine, 75012 Paris, France. ${ }^{13}$ Pôle Paris 11 Les Hôpitaux de Saint Maurice, 12-14 rue Val d'Osne, 94410 St Maurice, France. ${ }^{14}$ Service Hospitalo-universitaire, CH du Rouvray, 4 rue Paul Eluard, 76300 Sotteville-lès-Rouen, France. ${ }^{15}$ unité Inserm U1079 Faculté de médecine et de pharmacie, 76000 Rouen, France. ${ }^{16}$ ECEVE, UMR 1123 URCEco lle de France Hôtel Dieu, 1 place du Parvis de Notre Dame, 75004 Paris, France.

Received: 7 July 2018 Accepted: 11 September 2019 Published online: 08 November 2019

\section{References}

1. Carli $P$, et al. The French emergency medical services after the Paris and Nice terrorist attacks: what have we learnt? Lancet. 2017:390(10113):2735-8. 
2. Kessler RC, et al. Posttraumatic stress disorder in the National Comorbidity Survey. Arch Gen Psychiatry. 1995;52(12):1048-60.

3. APA Work Group on Psychiatric Evaluation, Silverman JJ, Fochtmann LJ, Vergare MJ, Anzia DJ, American Psychiatric Association, éditeurs. The American Psychiatric Association practice guidelines for the psychiatric evaluation of adults. Third edition. Arlington, VA: American Psychiatric Association; 2016. 164 p.

4. Chilcoat HD, Breslau N. Posttraumatic stress disorder and drug disorders: testing causal pathways. Arch Gen Psychiatry. 1998;55(10):913-7.

5. Breslau N. Epidemiologic studies of trauma, posttraumatic stress disorder, and other psychiatric disorders. Can J Psychiatry. 2002;47(10):923-9.

6. Brunet $A$, et al. Trauma reactivation under the influence of propranolo decreases posttraumatic stress symptoms and disorder: 3 open-label trials. J Clin Psychopharmacol. 2011;31(4):547-50.

7. Brunet $\mathrm{A}$, et al. Reduction of PTSD symptoms with pre-reactivation propranolol therapy: a randomized controlled trial. Am J Psychiatry. 2018. https://doi.org/10.1176/appi.ajp.2017.

8. Misanin JR, Miller RR, Lewis DJ. Retrograde amnesia produced by electroconvulsive shock after reactivation of a consolidated memory trace. Science. 1968;160(3827):554-5.

9. Przybyslawski J, Sara SJ. Reconsolidation of memory after its reactivation. Behav Brain Res. 1997;84(1-2):241-6.

10. Nader K, Schafe GE, Le Doux JE. Fear memories require protein synthesis in the amygdala for reconsolidation after retrieval. Nature. 2000;406(6797):722.

11. Besnard A, Caboche J, Laroche S. Reconsolidation of memory: a decade of debate. Prog Neurobiol. 2012;99(1):61-80.

12. Sara SJ. Retrieval and reconsolidation: toward a neurobiology of remembering. Learn Mem. 2000;7(2):73-84.

13. Nader K. Reconsolidation and the dynamic nature of memory. Cold Spring Harb Perspect Biol. 2015;7(10):a021782.

14. NICE. Post-traumatic stress disorder (PTSD): the management of PTSD in adults and children in primary and secondary careNICE Clinical Guidelines 26; 2005.

15. Brunet A, et al. Effect of post-retrieval propranolol on psychophysiologic responding during subsequent script-driven traumatic imagery in posttraumatic stress disorder. J Psychiatr Res. 2008;42(6):503-6.

16. Soeter M, Kindt M. An abrupt transformation of phobic behavior after a post-retrieval amnesic agent. Biol Psychiatry. 2015;78(12):880-6.

17. Lonergan $\mathrm{M}$, et al. Reactivating addiction-related memories under propranolol to reduce craving: a pilot randomized controlled trial. J Behav Ther Exp Psychiatry. 2016;50:245-9.

18. APA, American Psychiatric Association. Practice guideline for the treatment of patients with acute stress disorder and posttraumatic stress disorder. Am J Psychiatry. 2004;161(11 Suppl):3-31.

19. HAS. Affections psychiatriques de longue durée. Troubles anxieux graves: Haute Autorité de Santé; 2007.

20. Foa EB, Keane TM, Friedman MJ. Guidelines for treatment of PTSD. J Trauma Stress. 2000;13(4):539-88.

21. Ballenger JC, et al. Consensus statement update on posttraumatic stress disorder from the international consensus group on depression and anxiety. J Clin Psychiatry. 2004;65(Suppl 1):55-62.

22. Roberts NP, et al. Psychological therapies for post-traumatic stress disorder and comorbid substance use disorder. Cochrane Database Syst Rev. 2016; 4(Issue 4):CD010204

23. Marshall RD, et al. Efficacy and safety of paroxetine treatment for chronic PTSD: a fixed-dose, placebo-controlled study. Am J Psychiatry. 2001;158(12): 1982-8.

24. Marshall RD, et al. A controlled trial of paroxetine for chronic PTSD, dissociation, and interpersonal problems in mostly minority adults. Depress Anxiety. 2007;24(2):77-84

25. Tucker $P$, et al. Paroxetine in the treatment of chronic posttraumatic stress disorder: results of a placebo-controlled, flexible-dosage trial. J Clin Psychiatry. 2001:62(11):860-8.

26. Marks DM, et al. Paroxetine: safety and tolerability issues. Expert Opin Drug Saf. 2008;7(6):783-94

27. Cahill $L$, et al. Cognitive-behavioral therapy for adults. In: Foa EB, et al., editors. Effective treatment for PTSD. New York: The Guilford Press; 2009

28. Benedek DM, et al. Guideline watch: practice guideline for the treatment of patients with acute stress disorder and posttraumatic stress disorderAPA Practice Guidelines; 2009.
29. Bradley $\mathrm{R}$, et al. A multidimensional meta-analysis of psychotherapy for PTSD. Am J Psychiatry. 2005:162(2):214-27.

30. Kim SY. Efficacy versus effectiveness. Korean J Fam Med. 2013;34(4):227.

31. Ford I, Norrie J. Pragmatic trials. N Engl J Med. 2016;375(5):454-63.

32. Weathers FW, Litz BT, Herman DS, Huska JA, Keane TM, The PTSD Checklist (PCL). Reliability, validity, and diagnostic utility. San Antonio: Paper presented at the Annual Meeting of International Society for Traumatic Stress Studies; 1993.

33. Wilkins KC, Lang AJ, Norman SB. Synthesis of the psychometric properties of the PTSD checklist (PCL) military, civilian, and specific versions. Depress Anxiety. 2011:28(7):596-606.

34. Blanchard EB, et al. Psychometric properties of the PTSD checklist (PCL). Behav Res Ther. 1996;34(8):669-73.

35. Ventureyra VA, et al. The validation of the posttraumatic stress disorder checklist scale in posttraumatic stress disorder and nonclinical subjects. Psychother Psychosom. 2002;71(1):47-53.

36. Sheehan DV, et al. The Mini-international neuropsychiatric interview (M.I.N.I.): the development and validation of a structured diagnostic psychiatric interview for DSM-IV and ICD-10. J Clin Psychiatry. 1998;59 Suppl 20:22-33 quiz 34-57.

37. Brunet $A$, et al. The Peritraumatic distress inventory: a proposed measure of PTSD criterion A2. Am J Psychiatry. 2001;158(9):1480-5.

38. Jehel $L$, et al. Validation of the Peritraumatic distress Inventory's French translation. Can J Psychiatry. 2005;50(1):67-71.

39. Marmar CR, Weiss DS, Metzler TJ. The Peritraumatic dissociative experiences questionnaire. In: Wilson JP, Keane TM, editors. Assessing psychological trauma and posttraumatic stress disorder. New York: The Guilford Press; 1997. p. 412-28.

40. Birmes $P$, et al. Validation of the Peritraumatic dissociative experiences questionnaire self-report version in two samples of French-speaking individuals exposed to trauma. Eur Psychiatry. 2005;20(2):145-51.

41. Guardia, Dewi et al. Prediction of trauma-related disorders: a proposed cutoff score for the peritraumatic distress inventory. The primary care companion for CNS disorders. 2013;15(1):PCC.12101406.

42. Luo N, et al. A comparison of the scaling properties of the English, Spanish, French, and Chinese EQ-5D descriptive systems. Qual Life Res. 2013;22(8): 2237-43.

43. Herdman $M$, et al. Development and preliminary testing of the new fivelevel version of EQ-5D (EQ-5D-5L). Qual Life Res. 2011;20(10):1727-36.

44. Group, T.W. Development of the World Health Organization WHOQOL-BREF quality of life assessment. The WHOQOL group. Psychol Med. 1998;28(3): $551-8$

45. Baumann C, et al. The WHOQOL-BREF questionnaire: French adult population norms for the physical health, psychological health and social relationship dimensions. Rev Epidemiol Sante Publique. 2010;58(1):33-9.

46. Beecham J, Knapp M. Costing psychiatric interventions. In: Thornicroft G Brewin CR, Wing J, editors. Measuring mental health needs. London: The Royal College of Psychiatrists; 1992. p. 163-83.

47. Beecham J, Knapp M. Costing psychiatric interventions. In: Thornicroft G, editor. Measuring mental health needs. 2nd ed. London: The Royal College of Psychiatrists; 2001. p. 200-24.

48. Husereau D, et al. Consolidated health economic evaluation reporting standards (CHEERS) statement. BMC Med. 2013;11(1):80.

49. Zanello A, et al. Validation of the QFS measuring the frequency and satisfaction in social behaviours in psychiatric adult population. Encephale 2006;32(1 Pt 1):45-59.

50. Ventevogel $P$, et al. Properties of the Hopkins symptom Checklist-25 (HSCL25) and the self-reporting questionnaire (SRQ-20) as screening instruments used in primary care in Afghanistan. Soc Psychiatry Psychiatr Epidemiol. 2007;42(4):328-35

51. American Psychiatric Association. Diagnostic and statistical manual of mental disorders. 4th ed. Washington, DC; 2000.

\section{Publisher's Note}

Springer Nature remains neutral with regard to jurisdictional claims in published maps and institutional affiliations. 